\title{
A implicação da família no uso abusivo de drogas: uma revisão crítica
}

\author{
The family implication on the drug \\ abusive use: a critical review
}

M iriam Schenker 1

M aria Cecília de Souza M inayo 2

\footnotetext{
1 Núcleo de Estudos e Pesquisa em Atenção ao U so de Drogas/Uerj, Pós-Graduação em Saúde da Criança e da Mulher do Instituto Fernandes Figueira/Fiocruz. Av. Rui Barbosa, 716. 22250-020 Flamengo Rio de Janeiro RJ. mschenker00@hotmail.co $\mathrm{m}$

2 Centro Latino-

Americano de Estudos da Violência, Fiocruz.
}

Abstract This article presents a critical review of the litterature about the relationship between adolescence, family and drug abusive use. Discusses the importance of inserting the drugaddiction symptom in the familiar and sociocultural context for the understanding of its complexity. Family, school and peers are seen as the adolescent primary socialization sources. Family educational practices and parenting styles, with its three different types of parental control, are emphasized becau se they can either facilitate, or not, drug abusive use. The researches results point to the importance of engaging the family in the addict treatment and some studies enlarge the focus to engage multiple social contexts - family, friends, school, community and legal system - in the drug abusi ve adolescent treatment. The results of the researchs point to the family educational practices and parenting styles that ei ther facilitates, or not, drug abusive use, emphasizing the need to engage the family in the addict treatment.

Key words Adolescent, Family, Substance abuse, Treatment, Substance use disorder
Resumo Este artigo apresenta uma revisão crítica da literatura sobre a relação entre adolescência, família e uso abusivo de drogas. Discorre sobre a importância da inserção do sintoma drogadi cção no contexto familiar e sociocultural para o entendimento de sua complexidade. A família é vista como uma das fontes de socialização primária do adolescente, juntamente com a escola e o grupo de amigos. As práticas educativas e os estilos de criação da família, com seus três di ferentes ti pos de con trole parental, são ressal tados porque podem facilitar, ou não, o uso abusivo de drogas. 0 s resultados das pesqui sas apontam para a importância de se engajar a família no tratamento do adicto e al guns estudos ampliam o foco para en gajar contextos sociais múltiplos - família, amigos, escola, comunidade esistema legal - no tratamento do adolescente que faz uso abusivo de drogas.

Palavras-chave Adolescente, Família, Abuso de substância, Tratamento, Transtornos de uso de substância 


\section{Introdução}

Este artigo apresenta uma revisão crítica da literatura sobre o uso indevido e abusivo de drogas em geral e, de forma particular, por adolescentes. Focaliza, especificamente o lugar, o papel e a co-responsabilidade da família como instituição que elabora as relações primárias. A maioria dos textos revisados tem como objetivo socializar os conhecimentos; melhorar a qualidade do atendimento à população adicta; e subsidiar a implementação de políticas preventivas. A presente revisão abrange o período de 1995 a 2002 e ressalta como temas relevantes: a) o papel da família na tradução dos códigos culturais para os seus membros; b) a compreensão dos significados e condutas relativos ao uso de drogas; c) a parti cipação das práticas educativas da família na construção do comportamento de seus membros; d) a implicação do sistema familiar com o desenvolvimento do uso indevido de drogas; e) e o engajamento da família no tratamento.

Por família se entende uma instituição privada, passível, neste mundo pós-moderno, de vários tipos de arranjo, mas basicamente tendo a função de socialização primária das crianças e dos adolescentes. N este trabalho, parte-se do entendimento da adolescência como um período do ciclo vital em quea curiosidade por experiências novas e a troca e a influência do grupo de amigos são fundamentais. 0 uso das drogas aqui se inclui como fonte de socialização e como uma linguagem do adolescer e, quando acontece de forma abusiva, constitui-se num problema que pode repercutir em todo o processo posterior de vida do jovem.

Embora a atenção do adolescente esteja voltada para fora do lar e centrada nos grupos de colegas e amigos, para compreen dê-lo torna-se necessário inseri-lo no contexto familiar e sociocultural, pois a família - nuclear e extensa - integrada à cultura é que fornece as bases para o seu desenvolvimento. Ela éo lugar privilegiado da socialização primária (O etting \& Donnermeyer, 1998) cuja proposição principal éo asseguramento de comportamentos normalizados pelo afeto e pela cultura. Por isso, a família é fundamental no tratamento do uso abusivo dedrogas de seu adolescente.

0 engajamento da família no tratamento é enfatizado por aqueles pesquisadores que comungam das premissas do paradigma sistêmico, ponto de vista metodológico da presente revisão bibliográfica. Em relação às drogas, cada cultura tem diferentes formas de regulação (O etting \& Donnermeyer, 1998) de tal modo que seu uso pode ser requisitado, tolerado ou sancionado. Cabe ressaltar que 0 termo "adicção", - "adicto", "drogadicção" - é aqui empregado no seu sentido etimológico próprio: como um estado de submissão e dependência do indivíduo, com restrita vontade. Conceito relacional que engloba um indivíduo submisso, com pouca ou nenhuma consciência de seus atos. A compreensão de seus comportamentos se dará através da abordagem sistêmica que busca decodificar as premissas relacionais ignoradas pelo adicto (Sudbrack, 2001).

\section{Material e método}

As bases para a composição do presente artigo foram o Scielo e o M edline, entre os anos de 1995 e 2002, a partir das seguintes palavraschave: adolescente (adolescent); família (family); tratamento (treatment); abuso de substância (substance abuse); transtornos de uso de substância (substance use disorder).

Todo o material coletado, que constou de doze artigos na sua íntegra, nove resumos, três capítulos de livro e sete livros, foi devidamente fichado, analisado, comparado e avaliado quanto a sua contribuição para o objetivo já citado na introdução. $N$ a medida em que se foi observando que as questões de abuso surgidas na adolescência são plantadas desdea infância, pesquisou-se a relação entre infância, adolescência, família e abuso de drogas para, em seguida, investigar a necessidade de engajamento da família no tratamento deste sintoma.

O ponto de vista metodológico adotado neste trabal ho é o da teoria sistêmica cujas bases são: a) o sintoma do uso indevido ou abusivo da droga irrompe quando o contexto familiar e sociocultural oferecem condições de possibilidades para o seu surgimento e desenvolvimento; b) o comportamento de um indivíduo afeta e é afetado pelo comportamento do outro numa relação de circularidade enão delinearidade; c) é preciso trabalhar as interações familiares que dão suporte a padrões de desajuste na organização familiar. 


\section{D iscussão teórica}

Laqueille e colaboradores (1995) definem como dependentes os comportamentos dos drogadictos, pois el es são movidos pelo desejo poderoso, compulsivo de utilizar uma substância psicoativa, procura que invade, progressi vamente, toda a sua existência. Consideram a dependência uma patologia que tende a se tornar crônica, porque o adicto é ambivalente com relação à abstinência, nega a importância de sua dependência, recusa-se a admitir a gravidade da situação ao evitar suas angústias com o uso crescente das drogas, recorrendo, tardiamente, a cuidados especializados.

A idéia de que a dependência do adicto é uma patologia encaixa-se numa visão corrente do adicto como doente, em que se enfatiza mais a substância psicoativa do que a relação que o sujeito estabelece com a droga, em contextos múltiplos de relações - família, amigos, comunidade - , num determinado espaço sociocultural. As pesquisas descritas por H offmann \& Ceboneb (2002) mostram que os distúrbios no uso de drogas psicoati vas estão associados ao uso de drogas pelos adolescentes com baixa auto-estima, sintomas depressivos, eventos de vida estressantes, baixa coesão familiar eligação com amigos que consomem drogas.

Liddle e Dakof (1995b) chamam a atenção para a questão dos distúrbios provocados pelo uso abusivo de drogas serem uma ameaça à saúde pública, trazendo ônus considerável ao adicto e à sua família, pois comumente a adicção leva a perdas de empregos, rupturas familiares, instabilidade financeira e abuso físico e psicológico. A comunidade também é onerada, porque há vítimas de crimes e acidentes relacionados com as drogas, bem como al tos custos de encarceramento, no caso de substâncias ilegais, e tratamento.

As normas para os comportamentos sociais (O etting $\&$ D onnermeyer, 1998), incluindo-se aí o uso de drogas, são aprendidas predominantemente no contexto das interações com as fontes primárias de social ização que, na sociedade ocidental, são a família, a escola, e os amigos na adolescência. Cada uma das conexões das fontes primárias com o jovem envolve um vínculo que provêum canal para a comunicação de normas. Vínculos frágeis entre o jovem e essas fontes são fatores de risco para a instalação de desvios. Vínculos e ajustes saudáveis com a família e com a escola previnem a associação do jovem com as ditas "más companhias" na adolescência. Essas três fontes funcionam, para o jovem, como mediadoras das outras influências sociais vistas como secundárias: a religião, a mídia e a comunidade.

A família tem um papel importante na criação de condições relacionadas tanto ao uso abusivo de drogas pelo adolescente quanto aos fatores de proteção, funcionando igualmente como antídoto, quando o uso de drogas já estiver instalado (Liddle \& Dakof, 1995a). U ma vez que a família é um dos elos mais fortes dessa cadeia multifacetada que forma o uso abusivo de drogas instaurado na adolescência, muitas abordagens terapêuticas são "baseadas na família" e abrangem os fatores intrafamiliares, intra-individuais e socioculturais, de forma sistêmica.

Stanton \& Shadish (1997) referem-se a pesquisas que reforçam a idéia de que é preciso atingir as famílias e trabal har os vínculos entre seus membros, nos casos dos indivíduos adictos que querem sair dessa situação: da amostra pesquisada, de $60 \%$ a $80 \%$ moravam com os pais ou falavam diariamente pelo menos com um deles; e de 80 a $95 \%$ comunicavam-se com a família toda semana. Esse fenômeno foi reportado para os EU A, Porto Rico, Inglaterra, Itália e Tailândia. Portanto, o tratamento do adicto irá se beneficiar da inclusão do sistema familiar no trabal ho dos vínculos familiares, uma vez que os resultados das pesquisas desvelam a ligação estreita entre 0 adicto e seus familiares. Considera-se que tratar do dependente implica incluir o sistema familiar para trabal har os vínculos familiares.

Em relação ao universo familiar, diferentes autores, dentre os quais Stanton \& Shadish (1997), chegaram a al gumas conclusões convergentes: a) uma série de fatores familiares tem relação com o processo adictivo; b) 0 início do abuso de drogas e de overdoses pode ser precipitado pelo rompimento familiar, estresse e perdas; c) o modelo dos pais no que se refere ao uso de drogas e ál cool é importante; d) 0 abuso de drogas pode auxiliar a manutenção da homeostase familiar ou pode servir como uma forma de mobilizar os pais do adicto para tratamento; e) outros membros da família podem "facilitar" comportamentos que perpetuem 0 abuso de substância por um 
dos seus membros.

A família, uma das três fontes de socialização primária, ao construir vínculos saudáveis, comunica normas sociais salutares para os seus membros. $M$ as, famílias disfuncionais podem transmitir normas desviantes através do modelo de comportamento dos pais para os filhos. Os problemas de vinculação familiar advêm, em sua maioria, daqueles lares onde faltam habilidades para a criação dos fiIhos, reduzindo as chances de transmissão efetiva de normas sociais saudáveis.

0 estilo de criação dos pais (Liddle et al., 1998) é entendido como o clima emocional em que ocorre a socialização, uma vez que atos educativos específicos só terão eficácia no contexto de uma relação emocional apropriada. 0 estilo de criação refere-se às atitudes dos pais com relação aos filhos em situações diversas, podendo ocorrer sob a forma de três tipos de controle: autoritarismo, "com autoridade" (authoritative) e permissivo. 0 estilo de controle "com autoridade", que incorpora cordialidade e vigilância, está associado a uma adaptação positiva em diversas áreas de funcionamento dos pais e adolescentes ao longo do desenvolvimento. Trata-se de um esti lo de criação que inicia o indivíduo num sistema de reciprocidade, correlacionando-se de forma positiva com uma série de atitudes e comportamentos adolescentes incluindo-se 0 desempenho e o engajamento escolar.

No autoritarismo, os pais são autocráticos, muito exigentes e pouco responsivos ao seu adolescente, que, por sua vez, mostra-se obediente às regras, porém com baixa autoconfiança. No estilo permissivo, os pais podem ser indulgentes ou negligentes. No primeiro caso, há maior probabilidade de uso de drogas e desengajamento escolar; no segundo, em que falta aos pais tan to correspondência quanto exigência com relação aos filhos, estes apresentam problemas em várias áreas de funcionamento desde 0 autoconceito até a competência.

A gama de comportamentos dos pais que define a prática de criação dos adolescentes subdivide-se em categorias de monitoramento e supervisão; controle, consistência/coerência e rigor de disciplina; apoio e comunicação. Os adolescentes buscam naturalmente a sua independência em relação aos pais com o intui to de controlar e decidir acerca de sua vida. Os pais freqüentemente confundem essa atitude com rebelião, pois os filhos tendem, nessa etapa da vida, a questionar os seus val ores e opiniões.

Os amigos preenchem o vácuo do progressi vo desprendimento da tutela dos pais e são tratados, pelos jovens, como se fossem os primeiros em importância na sua vida. Compreender esses aspectos é fundamental para a transição saudável do adolescente, podendo ser negociados, com sucesso, numa relação estruturada no afeto eapoio mútuo entre pais e filhos. É na ausência de cordialidade, encorajamento, monitoramento e colocação suficiente de limites que os adolescentes têm dificuldade em fazer a transição entre a confiança primeira colocada nos pais para uma maior independência e foco cada vez maior nos amigos (Liddle et al., 1998).

O u seja, também os amigos e colegas de escola formam grupos de intimidade, influenciando, de forma marcante, a transmissão de normas na fase da adolescência. Por disporem delaços fortes e monitorarem, diretamente, atitudes e comportamentos de seus membros têm um papel muito importante nessa etapa da vida. Vários autores ( 0 etting \& Donnermeyer, 1998) têm encontrado forte ligação entre o uso de droga dos pares e o uso de droga pelo adolescente, porém, as investigações também evidenciam que os adolescentes não são cooptados por amigos anti-sociais, mas eles se tornam atraentes pelo fato de 0 meio familiar apresentar abundância de conflitos e desengajamento interpessoal. N esses casos, em geral, os pais não implementam práticas efetivas de educação que equilibram afeto, atenção elimites para os filhos.

$O$ estudo de H ogue\&Liddle (1999) focaliza os problemas comportamentais destrutivos da criança e do adolescente - especificamente agressão, desordem de conduta, uso de drogas, delinqüência e violência - como preocupações si gnificativas que comprometem 0 desenvolvimento saudável e colocam o jovem em risco.

0 estudo de Schmidt e colaboradores (1996) procurou saber se há relação entre a forma que o subsistema parental tem de criar os filhos em seus aspectos comportamentais, afetivos e cognitivos e o uso abusivo de drogas pelo adolescente. Essa prática foi dividida em oito categorias desenvolvidas a partir da literatura levando-se em conta: 0 apego, 0 estilo de vida familiar, relações, percepções sociais dos pais eobservações de atitudes dos genitores por meio de sessões gravadas em vi- 
deoteipe durante a terapia multidimensional defamília (Multidimensional Family Therapy - M DFT). As categorias foram: 1) disciplina assertiva de poder; 2 ) disciplina e comunicação positiva; 3) monitoramento e colocação de limites positivos; 4) monitoramento e colocação de limites negativos; 5) inconsistência interparental; 6 ) afeto negativo e desengajamento; 7) afeto positivo e compromisso; 8) inflexibilidade cognitiva (Schmidt et al. 1996). Já o estilo de criação foi classificado como "com autoridade", autoritarismo e conflituado-desengajado.

Schmidt e colaboradores (1996) analisaram o uso de drogas pelo adolescente através de relatório pessoal e análise de urina. 0 s referid os autores observaram 16 sessões de terapia de família pelo método multidimensional (M DFT). M ais de dois terços dos pais meIhoraram a forma de criar seus filhos e essa forma foi significativa para mudanças no comportamento do adolescente.

0 estudo de Schmidt e colaboradores corrobora a premissa de 0 etting e $D$ onnermeyer (1998) e de Alexander e Gwyther (1995), que também descrevem a família em seu papel de decodificação central dos processos sociais para os seus membros, evidenciando-se que 0 uso indevido de drogas passará pel o filtro da família.

Dakof e colaboradores (2001), numa pesquisa sobre o pré-tratamento, buscaram identificar fatores demográficos dos pais e dos adolescentes que influenciariam o engajamento dos jovens na terapêutica para o abuso de drogas. Duzentos e vinte e dois ad olescentes de famílias urbanas participaram da pesquisa em que a maconha era a droga de el eição. Os resultados mostraram que o engajamento no tratamento se relacionou, em ordem de importância, com as expectativas parentais positivas com relação à realização educacional de seus adolescentes; a observação acurada nos relatórios dos pais sobre o sintoma externo do jovem (comportamento delinqüente e agressivo); e os maiores níveis de conflito familiar percebido pelos jovens. Isso Ihes propiciou o forte argumento de que os adolescentes, filhos de pais que apresentaram a combinação de reconhecimento de problemas e a crença que seu filho pode ultrapassá-los através da realização na escola, engajaram-se no tratamento. As variáveis: renda familiar, sexo, status de grupo minoritário, status na justiça juvenil, estrutura familiar, idade da mãe, psico- patologia e características do tratamento não distinguiram o engajamento ou não do adolescente no tratamento. Os resultados sugerem que as percepções dos pais sobre os adolescentes são primordiais.

0 monitoramento e a supervisão dos pais em relação aos filhos são sinais das expectativas que têm de que se cumpram as normas familiares, protegendo-os de se associarem a pares desviantes, reduzindo, assim, a chance do uso de drogas ( 0 etting $\&$ D onnermeyer, 1998). A família, como já se mencionou, éa fonte primeira da maioria das cren ças e comportamentos relativos à saúde, transmitidos às crianças e aos adolescentes. 0 estudo de Hogue e Liddle (1999) fornece subsídios para se pensar a terapia de família com crianças como instrumento de prevenção de problemasfuturos.

Alexander e Gwyther (1995) entendem o uso abusivo de drogas como desordem biopsicossocial séria e complexa que gera problemas sistêmi cos em níveis variados, passando da célula para a família, a escola, o trabal ho e a sociedade. Por considerarem que o melhor tratamento deverá envolver o adolescente e sua família, os autores utilizam uma abordagem focal izada na família para a avaliação e administração de problemas referentes ao abuso de drogas. Ver a criança ou o adolescente como parte e não à parte do sistema familiar éum passo fundamental. 0 abuso de drogas tende a incluir outros membros da família e sabe-se que a adicção, por um dos pais, afeta todos os membros do grupo, podendo ser mais bem avaliado dentro do contexto familiar.

0 modelo utilizado por esses autores é semelhante ao M odelo de socialização primária de 0 etting $\&$ Donnermeyer (1998), porque, também para eles, as atitu des concernentes à drogadicção ou ao não envolvimento com el as são socialmente aprendidas, e éno seio da família que ocorrem as primei ras aprendizagens. Além disto, a família éo canal através do qual influências fundamentais se fazem notar pelo adolescente. Portanto, no caso de qualquer um do núcleo familiar fazer uso abusivo de drogas, recomenda-se que a família busque tratamento para que possa lidar com o impacto deste comportamento em seu funcionamento, tornando explícitos os mecanismos inconscientes desse processo autodestrutivo.

Em resumo, as abordagens que envolvem a unidade familiar nos problemas relativos ao 
de tratamento por uso abusi vo de drogas são consideradas mais ef etivas do que as abordagens de tratamento individual (Alexander \& Gwyther, 1995). 0 tratamento da família no caso de comportamentos anti-sociais na infância possui ampla base de suporte empírico (H ogue e Liddle, 1999). Na psicoterapia de crianças, o treinamento dos pais, através dos princípios de reforço do comportamento, inclui ensinamentos para a utilização tanto de disciplina quanto de comunicação de forma consistente. $\mathrm{H}$ oje, os diferentes tipos de tratamentos de família estão equipados para lidar com problemas reconhecidos como previsíveis do comportamento anti-social: monitoramento parental frouxo; vínculo e ligação pais-criança pobres; e hostilidade e conflito nesta relação.

Sabe-se, por experiência e através de pesquisas (Liddle et al., 1998), que os pais continuam influenciando seus filhos adolescentes ainda que, nesta fase, o grupo de amigos se torne muito importante. 0 conflito intenso entre pais e adolescentes, além de não ser a norma, dificulta o desenvolvimento da identidade desse ser em formação. A transição positiva para a adolescên cia se faz através da negociação de mudanças nas relações entre pais e filhos, em busca da autonomia. Q uando isto não acontece, 0 adolescente poderá se distanciar dos pais de forma hostil para conseguir manter o controle sobre sua independência. Desta forma, um dos principais objetivos das intervenções baseadas na família com adolescentes envolvidos com uso abusivo de drogas deve ser o da reconstrução do vínculo emocional dos pais em relação ao jovem, de forma $a$ atender às necessidades de ambos.

As estratégias de tratamento para o comportamento anti-social são as abordagens ecológicas, que lidam com contextos sociais múltiplos - família, amigos, escola, comunidade e sistema legal -, vistos como influenciando e sendo influenciados por tal comportamento. Ten do em vista que a família é a conexão dessas esferas sociais que se entrecruzam nas vidas das crianças e dos adolescentes (H ogue e Liddle, 1999), as terapias de família aparecem como possibilidade para o tratamento de problemas de abuso de drogas (Stanton \& Shadish, 1997) e detranstornos de comportamento.

Para Liddle e Dakof (1995b) existe uma distinção entre terapia de família e intervenções que envolvem a família. A primeira esta- belece uma conexão entre as relações familiares e a formação e o uso contínuo do abuso às drogas. As segundas são vistas como mais uma das áreas de intervenção e, além disto, envolvem a família somente em diferentes formas de auxílio ou de provisão de informação.

Há uma variação na perspectiva baseada na família (Liddle \& Dakof, 1995a) que vai desde model os com raízes comportamentais (behavioristas), passando pela terapia funcional da família advinda da teoria da aprendizagem social, até a terapia de família estrutural. O utra abordagem é a dos modelos integradores multissi stêmicos e multidimensionais, cujo foco é a ecologia do adolescente que faz uso abusivo de drogas, avaliando e intervindo na sua rede de influências - família, cultura de amigos, escola e sistema de justiça juvenil. Cada uma dessas partes é considerada um holon, tanto uma parte como o todo. Os indivíduos são considerad os organismos biopsicossociais bem como membros de outros sistemas como família, trabalho, grupos de amizade, comunidade, grupos étnicos. Seria um pensamento ou uma intervenção reducionista se somente uma dessas per spectivas fosse utilizada em detrimento das outras (Liddle, 1999).

\section{Conclusão}

As questões relativas ao uso abusivo de drogas pelo adolescente e a co-participação da família levantadas no presente artigo estão em consonância com a minha prática clínica como terapeuta de famílias adictas.

Observa-se que os pais, ou figuras substitutas, têm dificuldade em passar normas e limites para seus filhos. Há pouca habilidade para criá-los e educá-los, advindo daí uma má qualidade de vínculos familiares. Em relação aos jovens isso se manifesta na falta de assertividade e na ambigüidade com relação às leis e normas. Observa-se primeiro na conduta da criança e, posteriormente, do adolescente, que os limites do que lhes é concedi do estão esgarçados, havendo grande prejuízo para a sua formação e sérias conseqüências para a vida em família e em sociedade. As crianças e os adolescentes aceitam a autoridade dos pais - o estabel ecimento de regras claras e coerentes e a imposição de limites - quando há uma relação de confiança e afeto entre eles. 
Percebe-se que as famílias adictas buscam "terceirizar" suas responsabilidades com relação a seus filhos. Cultivam um tipo de comportamento irrespon sável, como se o dever de monitorar e supervisionar o comportamento dos adolescentes fosse al go mecânico, robótico, sem a necessidade de construção prévia da relação de confiança. Quanto mais a família é "desengajada" (M inuchin, 1974) nas suas relações interpessoais maior risco seus filhos correm de desenvolver comportamentos antisociais.

Premissas contemporâneas do paradigma sistêmico (Grandesso, 2000) indicam que as relações entre os indivíduos são co-construídas, processo que os tornam co-responsáveis pela criação, desenvolvimento e qualidade dessa relação. Crescer, passar de uma etapa do ciclo vital da família para a seguinte, implica negociações que resultam em modificações nas relações previamente estabel ecidas. Famílias disfuncionais geral mente têm pais ainda imaturos na forma de relacionar-se com os fiIhos. Inúmeras vezes, os pais são mais adolescentes que os filhos, pois se apresentam como amigos - peers - e não se posicionam como educadores, figuras de au tori dade, de confiança e de respeito para os adolescentes.

É verdade também que a família passa os seus valores e as suas crenças através das gerações, sendo a fonte primeira de acolhimento para os seus membros. Pelo fato de ser co-responsável pela formação dos indi víduos, a família está diretamente implicada no desenvolvimento saudável ou adoecido de seus membros.

É importante ressaltar que todas as intervenções baseadas na família (Schmidt et al., 1996) partem do princípio de que a mudança no indivíduo de um uso abusivo de drogas para a diminuição deste abuso, agregado a um funcionamento socialmente saudável, resulta da mudança no sistema familiar. A forma de criar os filhos é fundamental na constituição do indivíduo desde a infância até a adolescência. As práticas de criação características do mei o familiar de adolescentes que apresentam desordens de conduta e abuso de substância são: administração insatisfatória da família, criação omissa, disciplina e monitoramento parental inadequados, irritabilidade dos pais, processos familiares coercitivos. Por outro lado, dado o papel do meio familiar em proteger a criança de fatores de risco e a contribuição central da criação dos pais neste sentido, são dignas de nota as mudanças que hoje ocorrem na cultura dessa instituição de raiz, seja em relação à vida a dois, etambém aos diferentes arranjos que influenciam a convivência, os hábitos e costumes.

O pensamento sistêmico-ecológico, diversamente daquele que privilegia a dinâmica do indivíduo, focaliza no contexto das relações as questões vividas pelo ser humano, entendendo que todos os fenômenos se inter-relacionam, com maior ou menor intensidade, na teia que conforma a sua existência. Por isso, o comportamento do adolescente que faz uso abusivo de drogas é entendido no seu contexto de influências, no meio sociocultural em queestiver vivendo.

A terapia de família, então, passa a ser uma indicação permanente. Os tratamentos que envolvem a ecologia do jovem que usa droga de forma abusiva abrangem ainda melhor a complexidade do fenômeno da adicção em nossos dias. 0 diálogo intercontextual fornece a riqueza de condições de possi bilidade para a coconstrução de um contexto saudável para o adolescente. 


\section{Referências bibliográficas}

Alexander DE \& Gwyther RE 1995. Alcoholism in adolescents and their families - family-focused assessment and management. Pediatric Clinics of $\mathrm{N}$ orth America 42(1):217-234.

Blackson TC, Tarter RE \& M ezzich AC 1996. Interaction between childhood temperament and parental discipline practices on behavioral adjustement in preadolescent sons of substance abuse and normal fathers. The American Journal of D rug and Alcohol A buse 22(3):335-348.

Boyd SJ et al. 1999. Therelationship between parental history and substance use severity in drug treatment patients. The A merican Journal on Addictions/American A cademy of Psychiatrists in Alcoholism and Addictions 8(1):15-23.

Bucher R 1995. Prevenindo contra as drogas e DST/Aids: populações em situação de risco. CDIC, Brasília, 28pp.

Bukstein OG 2000. Disruptive behavior disorders and substance use disorders in adolescents. Journal of Psychoactive D rugs 32(1):67-79.

Cook LS 2001. Adolescent addiction and delinquency in the family system. Issues in M ental Health Nursing 22(2):151-157.

Dakof GA, Tejeda M \& Liddle HA 2001. Predictors of engagement in adolescent drug abuse treatment. Journal of the American A cademy of Child and Adolescent Psychiatry 40(3):274-281.

De M icheli D \& Formigoni M LOS 2002. Are reasons for the first use of drugs and family circumstances predictors of future use patterns? Addictive Behaviors 27(1):87-100.

Grandesso M A, 2000. Sobre a reconstrução do significado: uma análise epistemológica e hermenêutica da prática clínica. Casa do Psicólogo, São Paulo, 422pp.

Haley J, 1980. Leaving home: the therapy of disturbed young people. M CGraw-Hill, N ova York.

H offmann J \& Cerboneb FG 2002. Parental substance use disorder and the risk of adolescent drug abuse: an event history analysis. D rug and Alcohol Dependence 66(3):255-264.

Hogue A \& Liddle H A 1999. Family-based preventive intervention: an approach to preventing substance use and antisocial behavior. American Journal of O rthopsychiatry 69(3):278-293.

Kaminer Y 1999. Addictive disorders in adolescence. Psychiatric Clinics of N orth A merica 22(2):275278.

Kaufman E \& Kaufmann PN (eds.) 1979. Family therapy of drug and alcohol abuse. Gardner Press Inc., NovaYork.

Laqueille X, U ribé M \& Olié JP 1995. Aspects cliniques actuels des toxicomanies. La Revue du Practicien 45(11):1.359-1.363.

LiddleH A \& Dakof GA 1995a. Family-based treatment for adolescent drug use: state of the science, pp. 218-254. In E Rahdert \& D Czechowicz (eds.). Adolescent drug abuse: clinical assessment and therapeutic interventions. U.S. Department of $H$ ealth and Human Services. 156.

Liddle HA \& Dakof GA 1995b. Efficacy of family therapy for drug abuse: promising but not definitive. Journal of Marital and Family Therapy 21(4):511-543.

Liddle HA, Becker D \& Diamond GM 1997. Family therapy supervision, pp. 400-418. In CE Watkins,
Jr.(ed.). H andbook of psychotherapy supervision. John Wiley \& Sons, Nova York.

Liddle H A, Rowe C, Dakof G \& LykeJ 1998. Translating parenting research into clinical interventions for families of adolescents. Clinical Child Psychology and Psychiatry 3(3):419-443.

Liddle HA 1999. Theory development in a family-based therapy for adolescent drug abuse. Journal of Clinical Child Psychology 28(4):521-532.

Loveland-Cherry CJ 2000. Family interventions to prevent substance abuse: children and adolescents. Annual Review of N ursing Research 18:195-218.

M erikangas KR et al. 1998. Familial transmission of substance use disorders. Archives of General Psychiatry 55(11):973-979.

M inuchin S 1974. Families and family therapy. Harvard University Press, Cambridge.

M inuchin S 1984. Family kaleidoscope: images of violence and healing. H arvard U niversity Press, Cambridge.

O etting ER \& D onnermeyer JF 1998. Primary socialization theory: the etiology of drug use and deviance. Part I. Substance U se \& M isuse 33(4):995-1.026.

O etting ER, D onnermeyer JF, Trimble JE \& Beauvais F 1998. Primary socialization theory: culture, ethnicity, and cultural identification. The links between culture and substance use. Part IV. Substance Use \& Misuse 33(10):2.075-2.107.

Schmidt SE, LiddleHA \& Dakof GA, 1996. Changes in parenting practices and adolescent drug abuse during multidimensional family therapy. Journal of Family Psychology 10(1):12-27.

Schydlower M 2000. Adolescent substance use and abuse: current issues. Text M edicus 98(2):31-35.

Stanton MD \& Todd TC and Associates 1982. The family therapy of drug and abuses addiction. The Guilford Press, Nova York.

Stanton M D \& Shadish WR 1997. O utcome, attrition, and family-couples treatment for drug abuse: a meta-analysis and review of controlled, comparative studies. Psychological Bulletin 122:170-191.

Sudbrack M FO 2001. Terapia familiar sistêmica, pp. 403415. In SD Seibel \& Toscano Jr. (eds.). Dependência de drogas. Editora Atheneu, São Paulo.

Artigo apresentado em 3/9/2002

Aprovado em 3/10/2002

Versão final apresentada em 15/10/2002 